\title{
Local Structural Study of Ferroelectric Domain Boundaries Using STEM-CBED with a Fast Pixelated STEM Detector
}

\author{
Kenji Tsuda $^{1 *}$, Ryusuke Sagawa ${ }^{2}$, Hiroki Hashiguchi ${ }^{2}$ and Yukihito Kondo ${ }^{2}$ \\ 1. Frontier Institute for Interdisciplinary Research, Tohoku University, Sendai, Japan. \\ 2. JEOL Ltd., Tokyo, Japan. \\ * Corresponding author: k_tsuda@tohoku.ac.jp
}

Convergent-beam electron diffraction (CBED) has been applied for symmetry determination [1] and crystal structure and electrostatic potential analysis [2-4] of nano-scale local specimen areas. In order to examine specimens with local structural fluctuations, we proposed a combined use of scanning the transmission electron microscopy (STEM) and the CBED (STEM-CBED method [5]), which can be categorized into the 4D-STEM techniques. In the STEM-CBED method, CBED patterns are acquired pixel-by-pixel by scanning the convergent-beam electron probe with a sub-nanometer scan step. This method enables us to visualize nanometer-scale spatial distributions of local structures with picometerscale sensitivity to atom-displacements with the full use of CBED data.

Using the STEM-CBED, we investigated local structures of perovskite-type ferroelectric oxides. Nanoscale spatial fluctuations of the rhombohedral polarization clusters were visualized in the tetragonal and cubic phases of $\mathrm{BaTiO}_{3}$ and $\mathrm{KNbO}_{3}$ [5-8]. These results directly show the order-disorder-type characters in the structural phase transformations of these ferroelectrics, in contrast to the displacivetype model based on the soft-mode theory [9]. As a next step, it is very intriguing to apply this method to the local structure analysis of ferroelectric domain boundaries. However, long data acquisition time needed for the STEM-CBED experiments restricted its wider applications.

In the present study, we have acquired STEM-CBED data of ferroelectric domain boundaries of perovskite oxides using a transmission electron microscope JEM-ARM200F equipped with a fast pixelated STEM detector 4DCanvas [10]. The 4DCanvas detector with $264 \mathrm{x} 264$ pixels has a readout speed of 1000-4000 fps and a sensor with quantum efficiency close to $100 \%$, significantly reducing the acquisition time of STEM-CBED data.

Figure 1(a) shows a BF-STEM image of a 120 deg. ferroelectric domain boundary of the orthorhombic phase of $\mathrm{KNbO}_{3}$. Figures 1(b)-1(c) show CBED patterns obtained at positions A-C indicated by white dots in Fig. 1(a). Those STEM image and CBED patterns were extracted from a STEM-CBED data acquired at $256 \times 256$ positions, where the total exposure time was 66 sec. Crystal orientation changes around the domain boundary are clearly seen in the CBED patterns. Crystal orientation and polarization maps reconstructed from the STEM-CBED data will be shown in the presentation [11]. 


\section{References:}

[1] M Tanaka and K Tsuda, J. Electron Microsc. 60 (S1) (2011), p. S245.

[2] K Tsuda and M Tanaka, Acta Cryst. A55 (1999), p. 939.

[3] Y Ogata, K Tsuda and M Tanaka, Acta. Cryst. A64 (2008), p. 587.

[4] K Tsuda et al., Phys. Rev. B 81 (2010), p. 180102.

[5] K.Tsuda, A Yasuhara and M Tanaka, Appl. Phys. Lett. 102 (2013), p. 051913.

[6] K Tsuda and M Tanaka, Appl. Phys. Express 8, (2015), p. 081501.

[7] K Tsuda and M Tanaka, Appl. Phys. Express 9 (2016), p. 071501.

[8] K Tsuda and M Tanaka, Jpn. J. Appl. Phys. 56 (2017), p. 10PB09.

[9] W Cochran, Adv. Phys. 9 (1960), p. 387.

[10] R Sagawa et al., Microsc. Microanal. 23 (S1) (2017), p. 52.

[11] An author (KT) is grateful to Emer. Prof. Michiyoshi Tanaka and Prof. Masami Terauchi for their stimulating discussion and support.

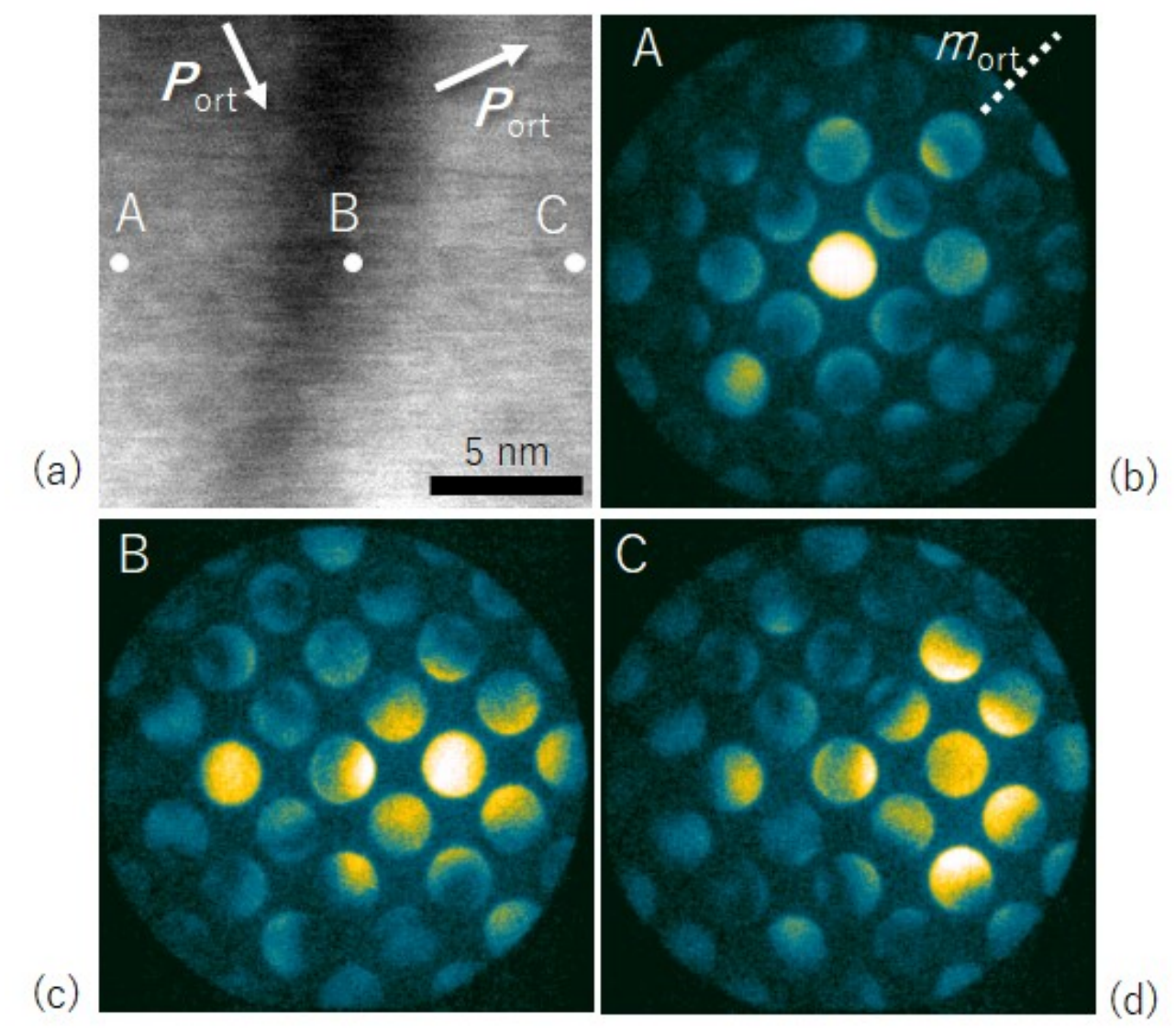

Figure 1. (a) BF-STEM image of $120 \mathrm{deg}$. ferroelectric domain boundary of the orthorhombic phase of $\mathrm{KNbO}_{3}$. (b)-(c) CBED patterns obtained at positions A-C indicated by white dots in Fig. (a) using the STEM-CBED method. 